\title{
A note on IFN-spaces
}

\author{
Reza Saadati ${ }^{1}$ and Choonkil Park ${ }^{2^{*}}$
}

\author{
${ }^{*}$ Correspondence: \\ baak@hanyang.ac.kr \\ ${ }^{2}$ Research Institute for Natural \\ Sciences, Hanyang University, Seoul, \\ 133-791, Korea \\ Full list of author information is \\ available at the end of the article
}

\begin{abstract}
Recently, Al-Fhaid and Mohiuddine (Adv. Differ. Equ. 2013:203, 2013) and Mohiuddine and Alghamdi (Adv. Differ. 2012:141, 2012) got some results in intuitionistic fuzzy normed spaces using ideas of intuitionistic fuzzy sets due to Atanassov and fuzzy normed spaces due to Saadati and Vaezpour. In this note, we show that the mentioned results follow directly from well-known theorems in fuzzy normed spaces. MSC: 54E40; 54E35; 54H25
\end{abstract}

Keywords: fuzzy normed space; intuitionistic fuzzy normed spaces; intuitionistic fuzzy set; topology

\section{Introduction}

Intuitionistic fuzzy normed spaces were investigated by Saadati and Park [1]. They introduced and studied intuitionistic fuzzy normed spaces based both on the idea of intuitionistic fuzzy sets due to Atanassov [2] and the concept of fuzzy normed spaces given by Saadati and Vaezpour in [3]. Next Deschrijver et al. [4] modified the concept of intuitionistic fuzzy normed spaces and introduced the notation of $\mathcal{L}$-fuzzy normed space. Recently, Al-Fhaid and Mohiuddine [5] and Mohiuddine and Alghamdi [6] got some results in intuitionistic fuzzy normed spaces. In this note we prove that the topology $\tau_{(\mu, v)}$ generated by an intuitionistic fuzzy normed space $(X, \mu, \nu, *, \diamond)$ coincides with the topology $\tau_{\mu}$ generated by the generalized fuzzy normed space $(X, \mu, *)$, and thus, the results obtained in [5] and [6] are immediate consequences of the corresponding results for fuzzy normed spaces.

\section{Preliminaries}

A binary operation $*:[0,1] \times[0,1] \rightarrow[0,1]$ is a continuous $t$-norm if it satisfies the following conditions:

(a) $*$ is associative and commutative,

(b) $*$ is continuous,

(c) $a * 1=a$ for all $a \in[0,1]$,

(d) $a * b \leq c * d$ whenever $a \leq c$ and $b \leq d$, for each $a, b, c, d \in[0,1]$.

Two typical examples of continuous $t$-norm are $a * b=a b$ and $a * b=\min (a, b)$.

A binary operation $\diamond:[0,1] \times[0,1] \rightarrow[0,1]$ is a continuous $t$-conorm if it satisfies the following conditions:

(a) $\diamond$ is associative and commutative,

(b) $\diamond$ is continuous,

(c) $a \diamond 0=a$ for all $a \in[0,1]$,

(d) $a \diamond b \leq c \diamond d$ whenever $a \leq c$ and $b \leq d$, for each $a, b, c, d \in[0,1]$. 
Two typical examples of a continuous $t$-conorm are $a \diamond b=\min (a+b, 1)$ and $a \diamond b=$ $\max (a, b)$.

In 2005, Saadati and Vaezpour [3] introduced the concept of fuzzy normed spaces.

Definition 2.1 Let $X$ be a real vector space. A function $\mu: X \times \mathbb{R} \rightarrow[0,1]$ is called a fuzzy norm on $X$ if for all $x, y \in X$ and all $s, t \in \mathbb{R}$,

$\left(\mu_{1}\right) \quad \mu(x, t)=0$ for $t \leq 0$;

$\left(\mu_{2}\right) x=0$ if and only if $\mu(x, t)=1$ for all $t>0$;

$\left(\mu_{3}\right) \mu(c x, t)=\mu\left(x, \frac{t}{|c|}\right)$ if $c \neq 0$;

$\left(\mu_{4}\right) \quad \mu(x+y, s+t) \geq \mu(x, s) * \mu(y, t)$

$\left(\mu_{5}\right) \quad \mu(x, \cdot)$ is a non-decreasing function of $\mathbb{R}$ and $\lim _{t \rightarrow \infty} \mu(x, t)=1$;

$\left(\mu_{6}\right)$ for $x \neq 0, \mu(x, \cdot)$ is continuous on $\mathbb{R}$.

For example, if $a * b=a b$ for $a, b \in[0,1],(X,\|\cdot\|)$ is normed space and

$$
\mu(x, t)=\frac{t}{t+\|x\|}
$$

for all $x, y, z \in X$ and $t>0$. Then $\mu$ is a (standard) fuzzy normed and $(X, \mu, \cdot)$ is a fuzzy normed space.

Saadati and Vaezpour showed in [3] that every fuzzy norm $(\mu, *)$ on $X$ generates a first countable topology $\tau_{\mu}$ on $X$ which has as a base the family of open sets of the form $\left\{B_{\mu}(x, r, t): x \in X, r \in(0,1), t>0\right\}$ where $B_{\mu}(x, r, t)=\{y \in X: \mu(x-y, t)>1-r\}$ for all $x \in X$, $r \in(0,1)$ and $t>0$.

\section{Intuitionistic fuzzy normed spaces}

Saadati and Park [1] defined the notion of intuitionistic fuzzy normed spaces with the help of continuous $t$-norms and continuous $t$-conorms as a generalization of fuzzy normed space due to Saadati and Vaezpour [3].

Definition 3.1 The 5-tuple $(X, \mu, v, *, \diamond)$ is said to be an intuitionistic fuzzy normed space if $X$ is a vector space, $*$ is a continuous $t$-norm, $\diamond$ is a continuous $t$-conorm, and $\mu, v$ are fuzzy sets on $X \times(0, \infty)$ satisfying the following conditions for every $x, y \in X$ and $t, s>0$ :

(a) $\mu(x, t)+v(x, t) \leq 1$,

(b) $\mu(x, t)>0$,

(c) $\mu(x, t)=1$ if and only if $x=0$,

(d) $\mu(\alpha x, t)=\mu\left(x, \frac{t}{|\alpha|}\right)$ for each $\alpha \neq 0$,

(e) $\mu(x, t) * \mu(y, s) \leq \mu(x+y, t+s)$,

(f) $\mu(x, \cdot):(0, \infty) \rightarrow[0,1]$ is continuous,

(g) $\lim _{t \rightarrow \infty} \mu(x, t)=1$ and $\lim _{t \rightarrow 0} \mu(x, t)=0$,

(h) $v(x, t)<1$,

(i) $v(x, t)=0$ if and only if $x=0$,

(j) $v(\alpha x, t)=v\left(x, \frac{t}{|\alpha|}\right)$ for each $\alpha \neq 0$,

(k) $v(x, t) \diamond v(y, s) \geq v(x+y, t+s)$,

(l) $v(x, \cdot):(0, \infty) \rightarrow[0,1]$ is continuous,

(m) $\lim _{t \rightarrow \infty} v(x, t)=0$ and $\lim _{t \rightarrow 0} v(x, t)=1$.

In this case $(\mu, v)$ is called an intuitionistic fuzzy norm. 
Example 3.2 Let $(X,\|\cdot\|)$ be a normed space. Denote $a * b=a b$ and $a \diamond b=\min (a+b, 1)$ for all $a, b \in[0,1]$ and let $\mu$ and $v$ be fuzzy sets on $X \times(0, \infty)$ defined as follows:

$$
\mu(x, t)=\frac{t}{t+\|x\|}, \quad v(x, t)=\frac{\|x\|}{t+\|x\|}
$$

for all $t \in \mathbf{R}^{+}$. Then $(X, \mu, v, *, \diamond)$ is an intuitionistic fuzzy normed space.

Saadati and Park proved in [1] that every intuitionistic fuzzy norm $(\mu, v)$ on $X$ generates a first countable topology $\tau_{(\mu, \nu)}$ on $X$ which has as a base the family of open sets of the form $\left\{B_{(\mu, v)}(x, r, t): x \in X, r \in(0,1), t>0\right\}$ where $B_{(\mu, v)}(x, r, t)=\{y \in X: \mu(x-y, t)>1-r, v(x-$ $y, t)<r\}$ for all $x \in X, r \in(0,1)$ and $t>0$.

Lemma 3.3 Let $(X, \mu, v, *, \diamond)$ be an intuitionistic fuzzy normed space. Then, for each $x \in X$, $r \in(0,1)$ and $t>0$, we have $B_{(\mu, v)}(x, r, t)=B_{\mu}(x, r, t)$.

Proof It is clear that $B_{(\mu, v)}(x, r, t) \subseteq B_{\nu}(x, r, t)$.

Now, suppose that $y \in B_{\mu}(x, r, t)$. Then $\mu(x-y, t)>1-r$, so, by condition (i) of Definition 3.1, we have

$$
1=\mu(x-y, t)+v(x-y, t)>1-r+v(x-y, t) .
$$

Hence $v(x-y, t)<r$, and consequently $y \in B_{(\mu, \nu)}(x, r, t)$. The proof is finished.

From Lemma 3.3, we deduce the following.

Theorem 3.4 Let $(X, \mu, v, *, \diamond)$ be an intuitionistic fuzzy normed space. Then the topologies $\tau_{(\mu, \nu)}$ and $\tau_{\mu}$ coincide on $X$.

\section{Competing interests}

The authors declare that they have no competing interests.

\section{Authors' contributions}

All authors carried out the proof. All authors conceived of the study, and participated in its design and coordination. All authors read and approved the final manuscript.

\section{Author details}

'Department of Mathematics, Iran University of Science and Technology, Tehran, Iran. ${ }^{2}$ Research Institute for Natural Sciences, Hanyang University, Seoul, 133-791, Korea.

Received: 26 December 2013 Accepted: 30 January 2014 Published: 11 Feb 2014

\section{References}

1. Saadati, R, Park, JH: On the intuitionistic fuzzy topological spaces. Chaos Solitons Fractals 27(2), 331-344 (2006)

2. Atanassov, KT: Intuitionistic fuzzy sets. Fuzzy Sets Syst. 20(1), 87-96 (1986)

3. Saadati, R, Vaezpour, SM: Some results on fuzzy Banach spaces. J. Appl. Math. Comput. 17(1-2), 475-484 (2005)

4. Deschrijver, G, O'Regan, D, Saadati, R, Vaezpour, SM: $\mathcal{L}$-Fuzzy Euclidean normed spaces and compactness. Chaos Solitons Fractals 42(1), 40-45 (2009)

5. Al-Fhaid, AS, Mohiuddine, SA: On the Ulam stability of mixed type QA mappings in IFN-spaces. Adv. Differ. Equ. 2013, $203(2013)$

6. Mohiuddine, SA, Alghamdi, MA: Stability of functional equation obtained through a fixed-point alternative in intuitionistic fuzzy normed spaces. Adv. Differ. Equ. 2012, 141 (2012)

10.1186/1687-1847-2014-63

Cite this article as: Saadati and Park: A note on IFN-spaces. Advances in Difference Equations 2014, 2014:63 